\title{
Retrospective comparison of high-resolution computed tomography of eosinophilic granulomatosis with polyangiitis with severe asthma
}

\author{
Xiaofeng Lin ${ }^{1,2 \#}$, Yuejiao Lin ${ }^{3,4 \#}$, Zhengdao Lai ${ }^{3,5 \#}$, Shushan $\mathrm{Wei}^{3}$, Minzhi Qiu ${ }^{3}$ Jianyu $\mathrm{Li}^{1}$, Qin Liu ${ }^{1}$, \\ Kian Fan Chung ${ }^{6}$, Qingsi Zeng ${ }^{1}$, Qingling Zhang ${ }^{3}$
}

${ }^{1}$ Department of Radiology, The First Affiliated Hospital of Guangzhou Medical University, Guangzhou, China; ${ }^{2}$ Department of Medical Imaging, State Key Laboratory of Oncology in South China, Collaborative Innovation Center for Cancer Medicine, Sun Yat-sen University Cancer Center, Guangzhou, China; ${ }^{3}$ Pulmonary and Critical Care Medicine, Guangzhou Institute of Respiratory Health, National Clinical Research Center for Respiratory Disease, National Center for Respiratory Medicine, State Key Laboratory of Respiratory Diseases, The First Affiliated Hospital of Guangzhou Medical University, Guangzhou, China; ${ }^{4}$ Respiratory Medicine, Shixing People's Hospital, Shaoguan, China; ${ }^{5}$ Department of Pulmonary and Critical Care Medicine, Dongguan People's Hospital, Southern Medial University, Dongguan, China; 'National Heart and Lung Institute, Imperial College London and Biomedical Research Unit, Royal Brompton and Harefield National Health Service Trust, London, UK

Contributions: (I) Conception and design: Q Zhang, Q Zeng; (II) Administrative support: Q Zhang, Q Zeng, KF Chung; (III) Provision of study materials or patients: X Lin, Y Lin, M Qiu; (IV) Collection and assembly of data: X Lin, Z Lai, S Wei, M Qiu; (V) Data analysis and interpretation: Z Lai, J Li, Q Liu; (VI) Manuscript writing: All authors; (VII) Final approval of manuscript: All authors.

\#These authors contributed equally to this work.

Correspondence to: Qingsi Zeng. Department of Radiology, The First Affiliated Hospital of Guangzhou Medical University, 151 Yanjiang Road, Yuexiu District, Guangzhou 510120, China. Email: 13660611505@163.com; Qingling Zhang. Pulmonary and Critical Care Medicine, Guangzhou Institute of Respiratory Health, National Clinical Research Center for Respiratory Disease, National Center for Respiratory Medicine, State Key Laboratory of Respiratory Diseases, The First Affiliated Hospital of Guangzhou Medical University, Guangzhou 510120, China. Email: zqling68@hotmail.com.

Background: Eosinophilic granulomatosis with polyangiitis (EGPA) is often misdiagnosed as severe asthma due to their similar clinical presentations. We compared the pulmonary radiologic features of EGPA to those of severe asthma by high-resolution computed tomography (HRCT) in order to early diagnose EGPA.

Methods: We retrospectively reviewed clinical records and HRCT findings of 96 patients with EGPA and 82 patients with severe asthma who were seen at our hospital from 2011 to 2017 . We used a semiquantitative grading system to evaluate radiological findings. A radiological only and a clinical-radiological model were used to differentiate EGPA from severe asthma.

Results: Bronchial wall thickening, air trapping, tree-in-bud opacities, bronchial mucus plugging, bronchiectasis, diffuse ground-glass opacities (GGOs), consolidation, and increased small vascular markings were more common in EGPA patients than in severe asthmatics $(\mathrm{P}<0.05)$. The gradings of GGO (grade $2 v s$. grade 1) and tree-in-bud opacities (grade $2 v s$. grade 0) were higher in EGPA patients than in severe asthmatics. The total image score of EGPA patients was significantly higher than that of severe asthmatics $(\mathrm{P}<0.05)$. In the radiological only and the clinical-radiological model, the area under the receiver operating characteristic (ROC) curves (AUCs) for the identification of EGPA and severe asthma were 0.904 [95\% confidence interval (CI): 0.860 to 0.948 ] and 0.974 (95\% CI: 0.955 to 0.993 ), respectively.

Conclusions: Lung HRCT scan is useful in differentiating EGPA from severe asthma. In patients with difficult-to-treat asthma, an HRCT scan of the thorax should be performed should there be features that raise the suspicion of EGPA. 
Keywords: Eosinophilic granulomatosis with polyangiitis (EGPA); severe asthma; high-resolution computed tomography (HRCT)

Submitted Apr 12, 2021. Accepted for publication Jun 16, 2021.

doi: $10.21037 / \mathrm{atm}-21-2243$

View this article at: https://dx.doi.org/10.21037/atm-21-2243

\section{Introduction}

Eosinophilic granulomatosis with polyangiitis (EGPA) is a systemic vasculitis characterized by concomitant symptoms of asthma, allergic rhinitis, and marked eosinophilia that was first described by Churg and Strauss in 1951 and was originally named Churg-Strauss syndrome (1). It was renamed EGPA during the 2012 Revised International Chapel Hill Consensus Conference (CHCC), with the name emphasizing its histopathological diagnostic criteria of tissue eosinophilia, necrotizing vasculitis, and extravascular eosinophil-rich granuloma (2). The lung is the earliest and most common site that is affected by EGPA (3-5). Involvement of the lungs in EGPA is very commonly seen on chest high-resolution computed tomography (HRCT), where abnormalities include ground-glass opacities (GGOs), consolidation, and bronchial wall thickening and/or small nodules (6-8).

Almost all EGPA patients demonstrate asthma-like symptoms at the early stage and require large doses of corticosteroids (9). Patients with EGPA are often misdiagnosed as having severe asthma which usually presents with bronchial wall thickening, and bronchiectasis on chest HRCT (10). Therefore, EGPA should be considered in the differential diagnosis of severe asthma. The findings of chest HRCT scans have previously been reported in 3 small series of patients with EGPA involving $7-31$ cases $(7,11,12)$. In the largest study thus far, which included 31 patients with EGPA, parenchymal GGOs and/ or consolidation were seen in $55 \%$ of participants, while bronchial wall thickening and/or bronchiectasis were observed in $50 \%$ of participants $(7,11,12)$. Differentiation of EGPA, especially prior to extra-pulmonary organ involvement, from cases of severe asthma is an important but often difficult exercise for the clinician as up to $96 \%$ of patients who subsequently develop EGPA can present with only the clinical features of severe asthma. It is also important to differentiate between EGPA and severe asthma as early as possible in the diagnostic pathway because the treatments of these conditions are not similar. In EGPA, the use of immunosuppressive therapy in addition to systemic corticosteroid therapy may be necessary (13).

In this study, we examined the chest HRCT abnormalities of 96 EGPA patients, prior to the onset of systemic involvement at a time when the localization of symptoms can be related to the airways and lungs only. We compared these HRCT abnormalities with those of patients with severe asthma in order to determine whether we can use the HRCT as a decision support tool to diagnose EGPA in patients who would otherwise have been diagnosed with severe asthma. We present the following article in accordance with the STARD reporting checklist (available at https://dx.doi.org/10.21037/atm-21-2243).

\section{Methods}

\section{Participants}

This study comprised an evaluation of the databases from the First Affiliated Hospital of Guangzhou Medical University from November 2011 to August 2017. All EGPA patients were diagnosed according to the diagnostic criteria proposed by the American College of Rheumatology (ACR) in 1990 (14), which stipulates the presence of at least 4 of 6 following criteria: (I) clinical diagnosis of asthma (II) blood eosinophilia of greater than $10 \%$ (III) neuropathy (IV) nonfixed pulmonary infiltrates (V) paranasal sinus abnormality and (VI) extravascular eosinophils. Patients were diagnosed with severe asthma if they met the criteria established by the European Respiratory Society (ERS) and American Thoracic Society (ATS) Task Force (15). The criteria were that the asthma required treatment with high dose inhaled corticosteroids plus a second controller and/or systemic corticosteroids to prevent it from becoming "uncontrolled" or that remains "uncontrolled" despite this therapy. Patients who were eventually diagnosed with other diseases during the follow-up period were excluded from this analysis. Based on the abovementioned inclusion/exclusion criteria, we screened 221 patients and included a total of 96 patients with EGPA of whom 80 were biopsy-confirmed with the 
presence of eosinophilic vasculitis in bronchial biopsies and 82 patients with severe asthma during the same time period. Laboratory analyses were performed within a week before patients were diagnosed and included peripheral blood eosinophil counts, and total serum immunoglobulin $\mathrm{E}$ (IgE) levels. A protocol was prepared before the study without registration. Approval of the protocols used in this study was granted by the Institutional Review Board at the First Affiliated Hospital of Guangzhou Medical University (No. 2018035). Written informed consent was retrospectively provided by each participant included in this study. All procedures performed in this study involving human participants were in accordance with the Declaration of Helsinki (as revised in 2013).

\section{Spirometry}

Forced vital capacity (FVC), forced expiratory volume in 1 second (FEV1), FEV1/FVC ratio, peak expiratory flow rate (PEFR), and mid-maximal expiratory flow (MMEF) were measured using a calibrated 2130 spirometer (Sensormedics, Anaheim, CA, USA). Pulmonary function tests were performed within a week before patients were diagnosed. At least 3 replicates were obtained for each measurement.

\section{HRCT}

We obtained HRCT images of the thorax from each patient upon his/her first admission to the hospital due to asthmalike symptoms, and the timing between HRCTs, pulmonary function tests, and blood counts were performed within 6 days. Images were obtained at full inspiration via 1 of the following 3 CT scanners. The Siemens Definition Somatom AS + 128-slice multidetector row CT (MRCT) scanner (Siemens, Erlangen, Germany) was adjusted to parameters of $120 \mathrm{kVp}, 120 \mathrm{mAs}, 0.6 \mathrm{~mm}$ collimation, and 1-2 mm intervals. The Siemens Perspective 128-slice MRCT scanner (Siemens, Erlangen, Germany) was adjusted to $110 \mathrm{kVp}$, $100 \mathrm{mAs}, 0.6 \mathrm{~mm}$ collimation, and 1-2 mm intervals. Finally, the Toshiba Aquilion 16-slice MRCT scanner (Toshiba, Tokyo, Japan) was adjusted to $120 \mathrm{kVp}, 200 \mathrm{mAs}, 1 \mathrm{~mm}$ intervals, and $1.0 \mathrm{~mm}$ collimation. In addition, 59 of the EGPA participants and 52 of those with severe asthma were also scanned at the end of maximal expiration.

All the CT images on our picture archiving and communication systems (PACS) system were independently reviewed by two independent chest radiologists (XFL and QSZ, with 5 and 30 years' experience in chest CT image interpretation, respectively), who were blinded to the diagnosis of the study subjects, and in the case of disagreement, consensus was reached by discussion. All HRCT images were assessed specifically for the patterns, location, and distribution of pulmonary lesions. Signs according to the glossary of terms recommended by the Fleischner society were as follows: (I) for airway: bronchial wall thickening, bronchiectasis, bronchial mucoid impaction, tree-in-bud opacities, and mosaic signs; and (II) for airspace signs: consolidation, single GGO, and diffuse GGOs (16). Other signs that were assessed included emphysema, interlobular septal thickening, atelectasis, increased small vascular markings, and mediastinal or hilar lymphadenopathy (16). Consolidation was defined as an area of opacification that obscured the underlying vessels; GGO was defined as a hazy increase in lung attenuation with no obscuration of underlying vessels; tree-in-bud signs were defined as nodular dilatation of centrilobular branching structures resembling a budding tree; emphysema was defined as a focal area of very low attenuation, usually without definable wall, that was surrounded by higherattenuation normal lung parenchyma; air trapping that was seen on end-expiration CT scans as parenchymal areas with less than the normal increase in attenuation and lack of volume reduction. Increased small vascular markings were defined as thickened or increased arteriole or venule bundles in the subpleural area that were no further than $10 \mathrm{~mm}$ into the pleura. We categorized EGPA radiologic abnormalities as airway, airspace, or mixed pattern, based on HRCT features of lung lesions (17). An airspace pattern was defined when consolidation or GGO were the predominant parenchymal abnormalities, whereas an airway pattern was defined when bronchial wall thickening, bronchiectasis, bronchial mucoid impaction, tree-in-bud opacities, and mosaic signs were the main abnormalities. A mixed pattern was defined as having radiological findings between airway and airspace patterns.

Consolidation, GGO, and tree-in-bud opacities were graded using the 6-level semi-quantitative system reported by Akira et al. (18). Briefly, these visual scores were defined as follows: grade 0 , none; grade 1 , less than $5 \%$; grade 2 , $5-24 \%$; grade 3, 25-49\%; grade 4, 50-74\%; and grade $5,>75 \%$ of lung parenchyma involvement (18). The anatomical extent of bronchial dilatation and bronchial mucoid impaction was scored based on the number of bronchopulmonary segments involved, as follows: grade 0 , none; grade 1, 1 segment; grade 2, 2-5 segments; grade 3, 6-9 segments; grade 4, 10-14 segments; and grade 5, 
Table 1 Clinical characteristics and lung function of participants

\begin{tabular}{|c|c|c|c|}
\hline Characteristics & EGPA $(n=96)$ & Severe asthma $(n=82)$ & $P$ value \\
\hline Gender, male: female & $49: 47$ & $35: 47$ & 0.265 \\
\hline Duration of asthma (years) & $4.00(1.08-8.00)$ & $5.50(2.00-12.00)$ & 0.028 \\
\hline Blood eosinophil count ( $\left.\times 10^{9} / \mathrm{L}\right)$ & $0.83(0.49-1.61)$ & $0.36(0.12-0.68)$ & $<0.001$ \\
\hline Total serum IgE (kU/L) & $459.00(182.00-1151.75)$ & 178.00 (91.65-383.75) & 0.041 \\
\hline FVC (\% predicted) & $84.29 \pm 17.55$ & $91.61 \pm 15.35$ & 0.004 \\
\hline FEV1 (\% predicted) & $63.57 \pm 22.61$ & $68.69 \pm 20.48$ & 0.121 \\
\hline FEV1/FVC (\%) & $62.42 \pm 16.65$ & $67.58 \pm 17.38$ & 0.047 \\
\hline
\end{tabular}

Data are presented as mean $\pm \mathrm{SD}$, or median (IQR). EGPA, eosinophilic granulomatosis with polyangiitis; IgE, immunoglobulin E; FVC, forced vital capacity; FEV1, forced expiratory volume in the first second; PEFR, peak expiratory flow rate; MMEF, mid-maximal expiratory flow; SD, standard deviation; IQR, interquartile range.

$\geq 14$ segments. The maximum score for each level was 5 and the total score could range from 0 to 25 .

\section{Statistical analysis}

Statistical analysis was performed using the software SPSS 22.0 (SPSS Inc., Chicago, IL, USA). Normally distributed data were expressed as the mean \pm standard error $(\mathrm{SE})$, while data that were non-normally distributed are expressed as the median and interquartile range (IQR). Differences between two groups were compared using Student $t$-tests or Mann-Whitney U non-parametric unpaired analysis, whereas multi-group comparisons were performed via analysis of variance (ANOVA) or Kruskal-Wallis nonparametric unpaired analysis. Differences in frequencies of abnormal HRCT findings were tested using chi-square test. A P value $<0.05$ was considered statistically significant.

The HRCT findings (including single GGO, diffuse GGO, consolidation, bronchial wall thickening, tree-inbud opacities, bronchial mucoid impaction, bronchiectasis, increased small vascular markings, interlobular septal thickening, emphysema, and atelectasis) were correlated with peripheral-blood eosinophil counts, peripheral-blood eosinophil percentages in blood, total serum $\mathrm{IgE}$ levels, FVC, FEV1, FEV1/FVC, PEFR, and MMEF. Spearman correlation analysis was used for correlation analysis.

Cohen's kappa coefficient $(\kappa)$ was used to determine the inter-observer agreement for the assessment of radiologic features and the semi-quantitative system. Inter-observer agreement was considered as slight [interclass correlation coefficient (ICC): 0.11-0.40], fair (ICC: 0.41-0.60), moderate (ICC: $0.61-0.80$ ), and good (ICC: $\geq 0.81-1.00$ ).

A logistic regression model was used to identify the optimal radiologic and clinical features that can differentiate EGPA from severe asthma. We combined the clinical guidance and univariate logistic regression by forward stepwise procedure to select the relevant variables and carried out multiple logistic regression. Then, we presented the results from two models: radiological alone, and a combined clinical-radiological. The performance of these models was assessed with respect to discrimination using the area under the curve (AUC) of receiver operating characteristic (ROC) analysis.

\section{Results}

\section{Clinical characteristics and lung function}

Clinical characteristics and lung function of participants with EGPA and severe asthma are summarized in Table 1. Among the EGPA participants, 93 (97\%) had a history of asthma. The duration of disease was significantly shorter in EGPA participants than that of severe asthmatics. The peripheral blood eosinophil count and total $\operatorname{IgE}$ level were 
Table 2 Participants with positive-HRCT findings in EGPA and in severe asthma

\begin{tabular}{|c|c|c|c|}
\hline HRCT findings & EGPA (n=96), n [\%] & Severe asthma $(\mathrm{n}=82), \mathrm{n}[\%]$ & $P$ value \\
\hline Bronchial wall thickening & 93 [97] & $69[84]$ & 0.003 \\
\hline Air trapping & $58 / 59[98]^{*}$ & $42 / 52[81]^{\#}$ & 0.002 \\
\hline Tree-in-bud opacities & 60 [63] & $12[15]$ & $<0.001$ \\
\hline Bronchiectasis & $38[40]$ & 5 [6] & $<0.001$ \\
\hline Mosaic perfusion & 9 [9] & $3[4]$ & 0.129 \\
\hline \multicolumn{4}{|l|}{ Airspace signs } \\
\hline Diffused GGOs & $71[74]$ & 15 [18] & $<0.001$ \\
\hline \multicolumn{4}{|l|}{ Other signs } \\
\hline Increased small vascular markings & $44[46]$ & 2 [2] & $<0.001$ \\
\hline Emphysema & 35 [36] & $17[21]$ & 0.021 \\
\hline Mediastinal or hilar lymphadenopathy & 22 [23] & $11[13]$ & 0.074 \\
\hline Interlobular septal thickening & 18 [19] & $2[3]$ & 0.001 \\
\hline Atelectasis & $11[11]$ & $1[1]$ & 0.007 \\
\hline
\end{tabular}

Data are presented as $n$ (\%). *, 59 of the EGPA participants underwent HRCT scans at both inspiration and expiration phases. *, 52 participants with severe asthma underwent HRCT scans at both inspiration and expiration phases. HRCT, high-resolution computed tomography; EGPA, eosinophilic granulomatosis with polyangiitis; GGO, ground-glass opacity.

significantly higher in EGPA patients compared with those in severe asthmatics. The FVC (\% predicted) and FEV1/ FVC ratio were lower in participants with EGPA compared with those of severe asthma participants.

\section{HRCT findings}

The frequencies of HRCT abnormalities in EGPA and severe asthma patients are listed in Table 2. Bronchial wall thickening and air trapping were the most common HRCT features observed in both EGPA and severe asthma participants but were more common in EGPA patients (Figure $1 A, B, C)$ than in those with severe asthma (Figure $1 D, E$ ). Other abnormalities seen in HRCT of EGPA participants included tree-in-bud opacities (Figure $1 F$ ), bronchial mucus plugging, bronchiectasis, diffuse GGOs (Figure 1G), consolidation (Figure 1H,I), increased small vascular markings (Figure 17), emphysema, interlobular septal thickening, and atelectasis, all of which were more common in EGPA participants than in those with severe asthma. The inter-observer agreement of the semi-quantitative grading method was fairly good, with the $\kappa$-values for all the radiologic variables assessed being 0.81-0.88. Using a semi-quantitative grading method, we found that the median grades of GGOs and tree-in-bud opacities were both grade 2 in EGPA participants, while the median grades of GGOs and tree-in-bud opacities were grade 1 and grade 0 , respectively, in those with severe asthma. The median total image score of participants with EGPA was higher than that of those with severe asthma [7.00 (3.25-10.00) vs. 1.00 (0-3.00), $\mathrm{P}<0.05$; Figure 2].

\section{CT-based clinical comparisons}

Airway, airspace, and mixed patterns of EGPA were observed in $59 \%(\mathrm{n}=57), 19 \%(\mathrm{n}=18)$, and $22 \%(\mathrm{n}=21)$ of EGPA participants, respectively (Table 3). Both PEF (\% predicted) and MMEF (\% predicted) were higher in 


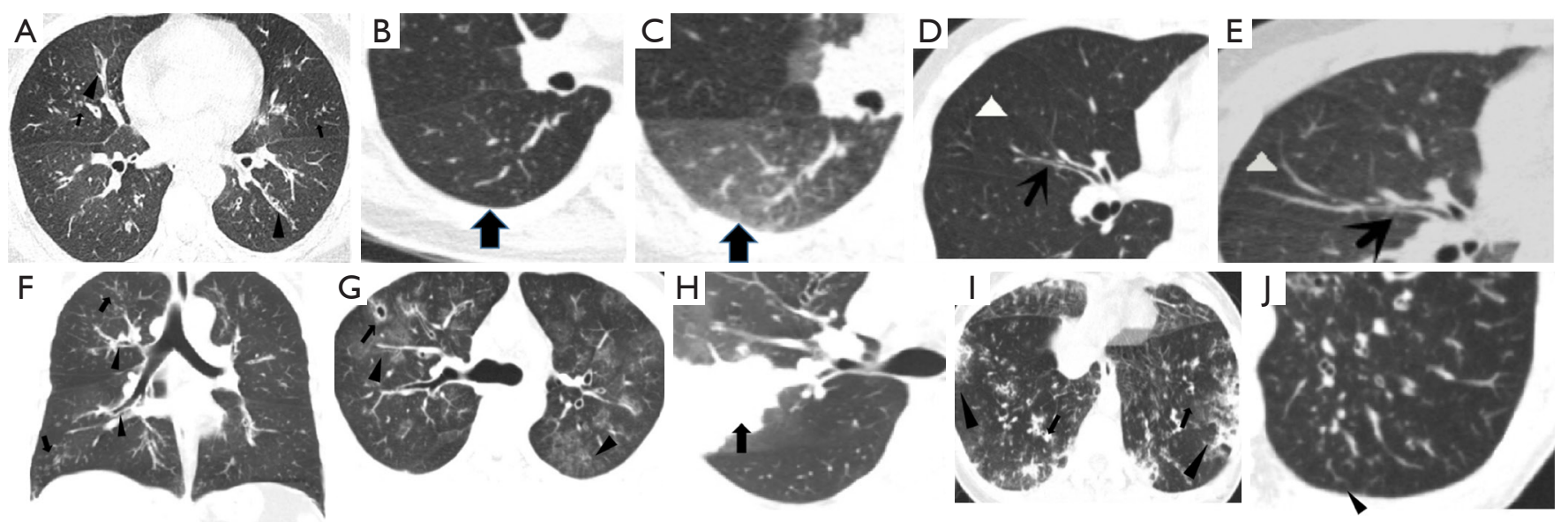

Figure 1 Imaging findings for EGPA and severe asthma. (A,F) Forty-two-year-old female patient with EGPA. The HRCT shows diffuse bronchial wall thickening (triangle) and tree-in-bud opacities (arrows) in both (A) axial and (F) coronal views. (B,C) The HRCT scan at inspiratory and full expiration of a 54-year-old female patient with EGPA revealing diffuse air trapping in expiration phase (arrows). (D,E) An HRCT image of a 39-year-old male patient with severe asthma shows bronchial wall thickening (arrows) and HRCT scan at full expiration reveals diffuse air trapping (triangle). (G) An HRCT image of a 29-year-old male patient with EGPA showing multiple patchy GGOs (triangle), with diffuse bronchial wall thickening, the right upper anterior segment of the lung bronchiectasis (arrows). (H) The HRCT image of a 29-year-old male patient with EGPA showing a large area of patchy consolidation (arrows) accompanied by GGO in the right lobe of the lung. (I) The HRCT image of a 43-year-old female patient with EGPA shows ill-defined distribution of consolidation (triangle) with GGOs in their periphery in the subpleural region and diffuse bronchial wall thickening (arrows). (J) An HRCT image of a 45-year-old male patient with EGPA showing increased small vascular markings (triangle) in the subpleural area. EGPA, eosinophilic granulomatosis with polyangiitis; HRCT, high-resolution computed tomography; GGO, ground-glass opacity.

participants with the airspace pattern of EGPA than in those with airway or mixed patterns $(\mathrm{P}<0.05)$. Median values of total $\mathrm{IgE}$ differed among participants with airway, airspace, and mixed patterns $(383.00,544.50$, and $709.00 \mathrm{kU} / \mathrm{L}$, respectively; $\mathrm{P}<0.05)$. By contrast, compared with that in EGPA participants, a lower percentage of those with severe asthma showed airspace (0\%) and mixed patterns (7\%), whereas there was a higher percentage of severe asthmatics with an airway pattern (93\%). Correlation analysis of imaging signs with clinical characteristics and lung function are shown in Figure 3. The most significant correlation was between the peripheral blood eosinophil count (\%) and diffuse GGOs, but this was only $\mathrm{P}=0.360$.

\section{Radiological and clinical-radiological models}

As shown in Tables 4,5, bronchial mucoid impaction, diffuse GGOs, and increased small vascular markings were incorporated into the radiological model. Bronchial wall thickening, bronchial mucoid impaction, increased small vascular markings, atelectasis, total serum IgE, FVC (\% predicted), FEV1/FVC (\%), and PEFR (\% predicted) were incorporated into the clinical-radiologic model. Finally, multi-factorial combined ROC curve analysis showed that the radiological model for differential diagnosis yielded a $74.5 \%$ sensitivity and $88.6 \%$ specificity, with an AUC of $90.4 \%$ [95\% confidence interval (CI): 0.860 to 0.948 ]. The clinical-radiological model for differential diagnosis yielded a $94.7 \%$ sensitivity and $88.6 \%$ specificity, with an AUC of 0.974 (95\% CI: 0.955 to 0.993 ) (Figure 4).

\section{Discussion}

Most commonly, EGPA affects the lungs and airways and its clinical features usually consist of rhinitis- and asthma-like symptoms that cannot be easily controlled by corticosteroids (9). Due to these presentations, many patients with EGPA are initially misdiagnosed as severe asthma. In the present retrospective study, we compared HRCT scans of 96 EGPA patients with those of 82 patients with severe asthma. These participants fell into these two categories, EGPA and severe asthma, according to the agreed upon definitions $(14,15)$. 
A
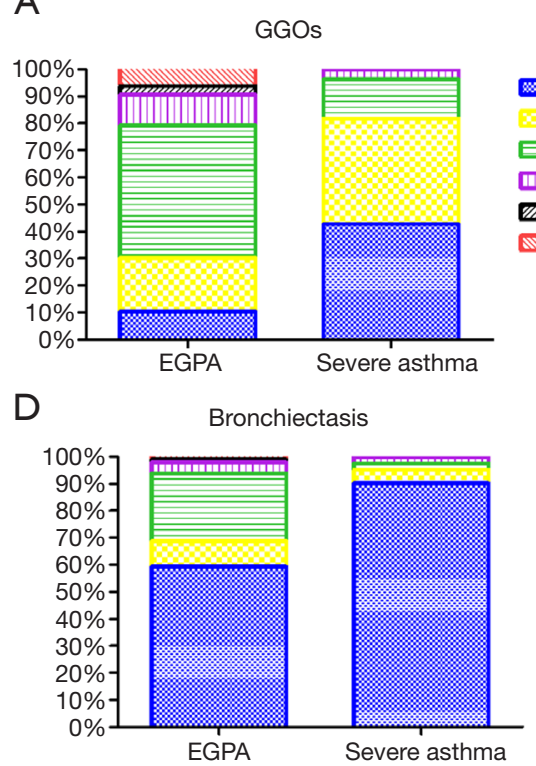

B

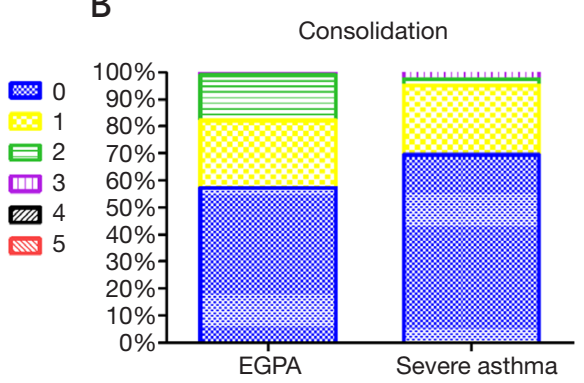

$\mathrm{E}$

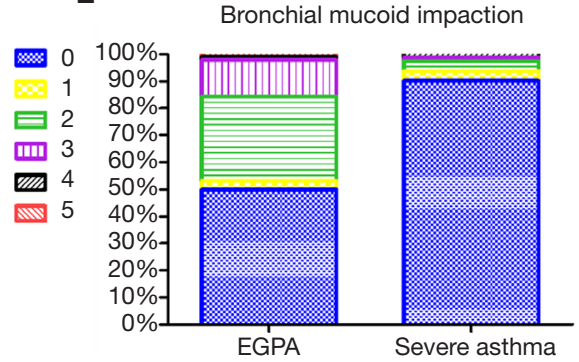

C

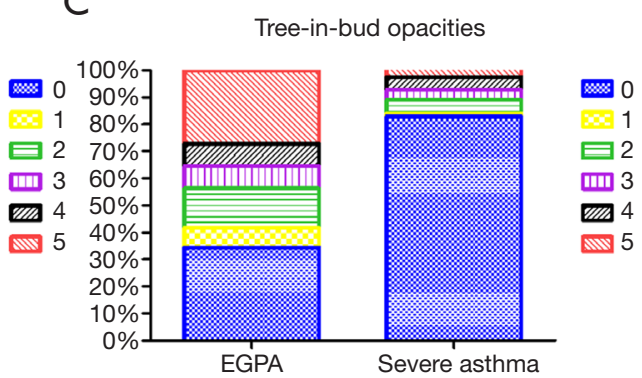

$\mathrm{F}$

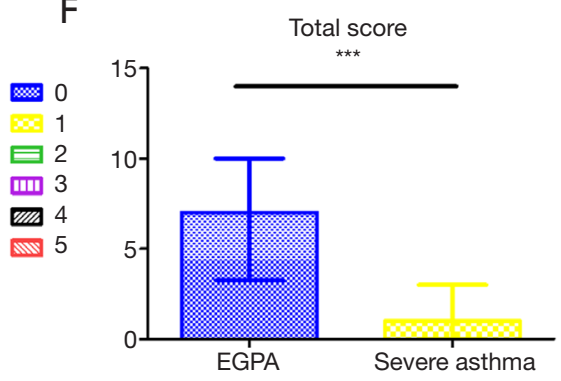

Figure 2 Imaging scoring of GGO consolidation, tree-in-bud opacities, bronchiectasis, bronchial mucoid impaction and total score in EGPA and severe asthma. (A) Imaging scoring of GGO in EGPA and severe asthma. (B) Imaging scoring of consolidation in EGPA and severe asthma. (C) Imaging scoring of tree-in-bud opacities in EGPA and severe asthma. (D) Imaging scoring of bronchiectasis in EGPA and severe asthma. (E) Imaging scoring of bronchial mucoid impaction in EGPA and severe asthma. (F) Total imaging scoring in EGPA and severe asthma. The difference in total scores between the two groups was significant $(* * *, \mathrm{P}<0.001)$. GGO, ground-glass opacity; EGPA, eosinophilic granulomatosis with polyangiitis.

Table 3 Lung function and blood characteristics of different HRCT patterns in EGPA

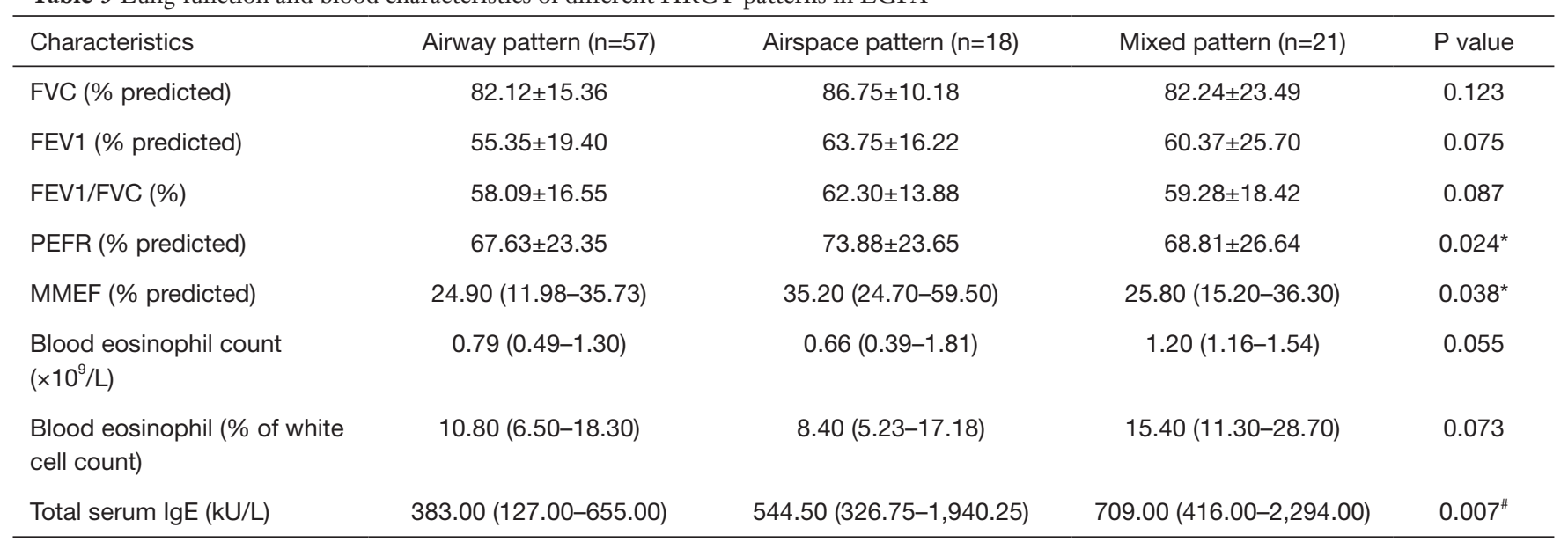

Data are presented as mean $\pm \mathrm{SD}$, or median (IQR). ${ }^{*}$, airspace and mixed patterns differed significantly according to the Bonferroni test. Other between-group differences were not statistically significant. \#, between-group differences among HRCT patterns were statistically significant according to the Bonferroni test. HRCT, high-resolution computed tomography; EGPA, eosinophilic granulomatosis with polyangiitis; FVC, forced vital capacity; FEV1, forced expiratory volume in the first second; PEFR, peak expiratory flow rate; MMEF, midmaximal expiratory flow; IgE, immunoglobulin E; SD, standard deviation; IQR, interquartile range. 


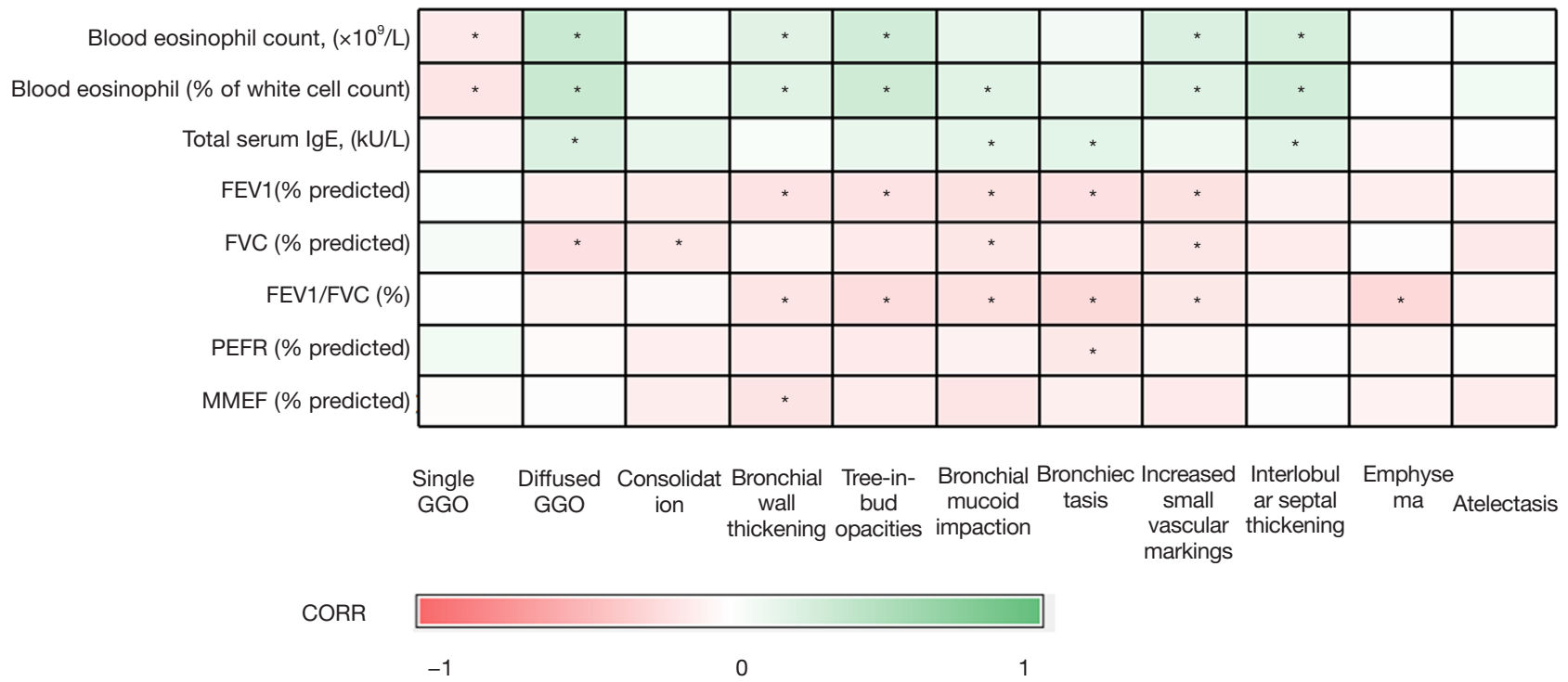

Figure 3 Correlations between imaging findings and clinical characteristics and lung function are shown on this heat map. Green is associated with a positive correlation while red is associated with the negative correlation. The darker the color, the stronger relationship (*, P<0.05). GGO, ground-glass opacity; IgE, immunoglobulin E; FEV1, forced expiratory volume in the first second; FVC, forced vital capacity; PEFR, peak expiratory flow rate; MMEF, mid-maximal expiratory flow.

Table 4 Multivariate logistic regression analysis of radiologic findings for differential diagnosis

\begin{tabular}{|c|c|c|c|c|c|c|}
\hline HRCT findings & $\mathrm{B}$ & SE (b) & Wald $\chi^{2}$ & $P$ value & OR & $95 \% \mathrm{Cl}$ for OR \\
\hline Tree-in-bud opacities & -0.815 & 0.536 & 2.311 & 0.128 & 0.443 & $0.155-1.266$ \\
\hline Bronchial mucoid impaction & -1.181 & 0.522 & 5.116 & 0.024 & 0.307 & $0.110-0.854$ \\
\hline Bronchiectasis & -0.875 & 0.662 & 1.744 & 0.187 & 0.417 & $0.114-1.527$ \\
\hline Consolidation & 0.138 & 0.536 & 0.066 & 0.797 & 1.148 & $0.402-3.279$ \\
\hline Single GGO & -0.843 & 0.602 & 1.962 & 0.161 & 0.430 & $0.132-1.400$ \\
\hline Increased small vascular markings & -2.790 & 0.821 & 11.564 & 0.001 & 0.061 & $0.012-0.307$ \\
\hline Emphysema & -0.494 & 0.515 & 0.918 & 0.338 & 0.610 & $0.222-1.675$ \\
\hline
\end{tabular}

$\mathrm{B}$, regression coefficient; SE (b), standard error of regression coefficient; Wald $\chi^{2}$, chi-square value; OR, odds ratio; Cl, confidence interval; GGO, ground-glass opacity.

Airway abnormalities (particularly bronchial wall thickening and tree-in-bud opacities), parenchymal opacification (GGOs and consolidations), and increased small vascular markings were more common in participants with EGPA than in severe asthmatics. The total image score of participants with EGPA was significantly higher than that of those with severe asthma. Finally, using the radiological only and the combined clinical-radiological models, the AUCs for the identification of EGPA and severe asthma were $90.4 \%$ and $97.4 \%$, respectively, supporting the 
Table 5 Multivariate logistic regression analysis of radiologic-clinical characteristics for differential diagnosis

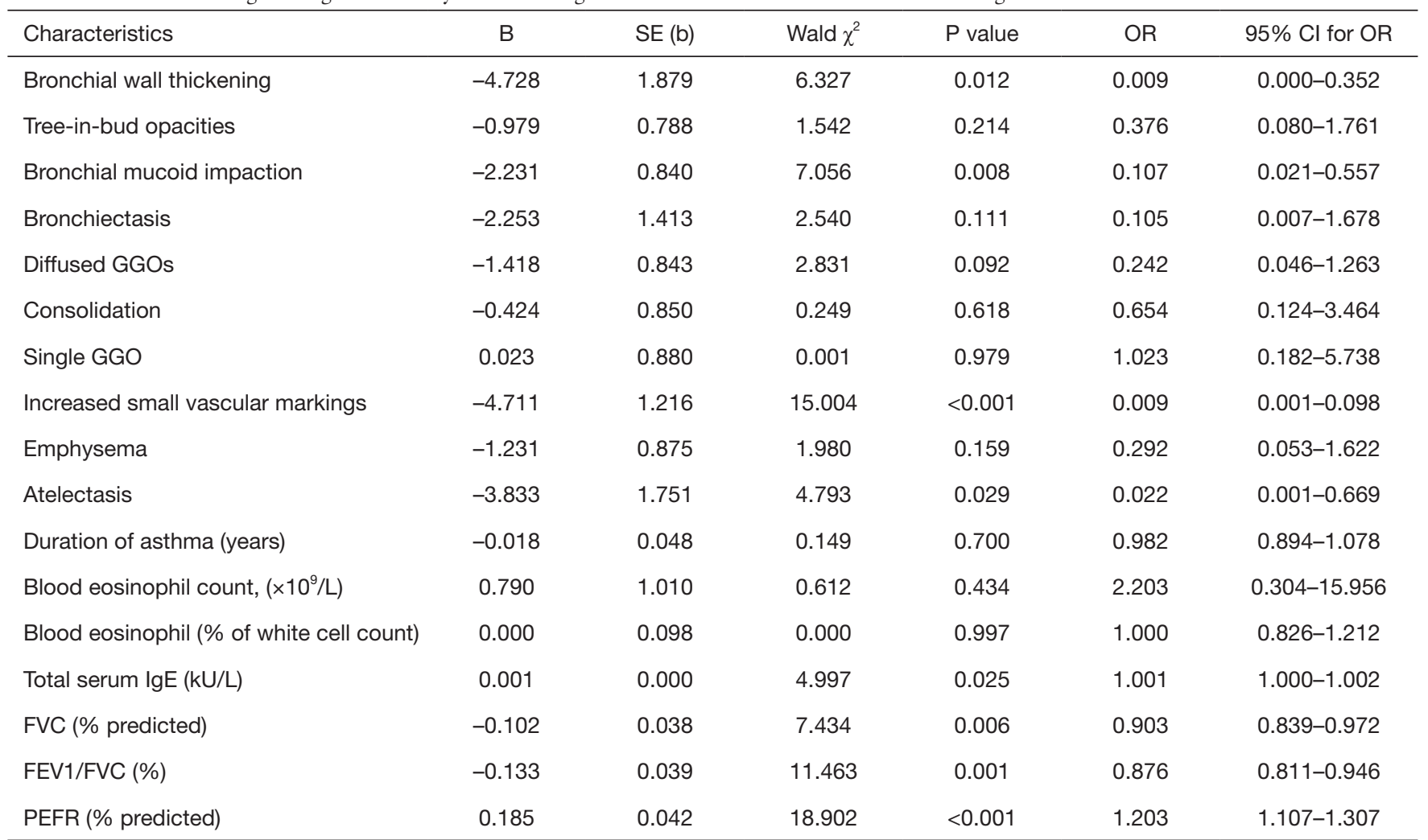

$\mathrm{B}$, regression coefficient; SE (b), standard error of regression coefficient; Wald $\chi^{2}$, chi-square value; OR, odds ratio; Cl, confidence interval; GGO, ground-glass opacity; IgE, immunoglobulin E; FVC, forced vital capacity; FEV1, forced expiratory volume in the first second; PEFR, peak expiratory flow rate.

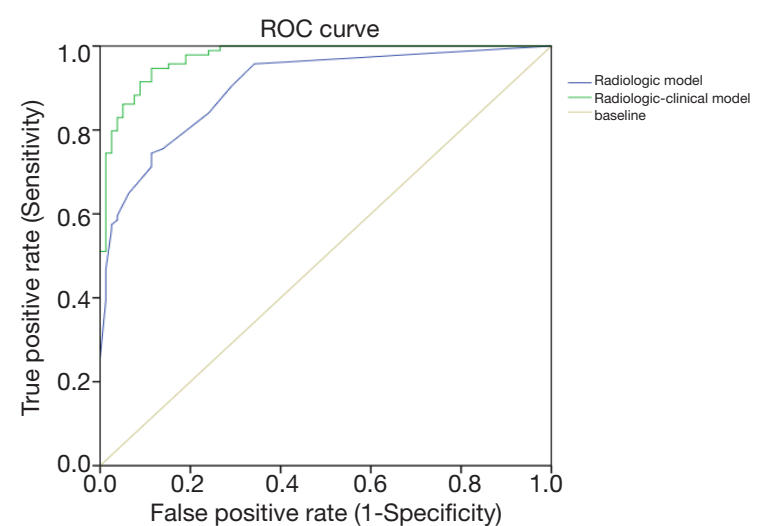

Figure 4 ROC curve for logistic regression of radiologic features alone (AUC $=0.906$ ) and of combined clinical and radiologic features ( $\mathrm{AUC}=0.937$ ). ROC, receiver operating characteristic; AUC, area under the ROC curve. usefulness of performing an HRCT scan of the lungs to differentiate EGPA from severe asthma. Interestingly, using only the radiological features was most contributory for supporting the diagnosis of EGPA, while the addition of clinical features provided some further sensitivity for the diagnosis.

The most commonly-reported HRCT manifestations in EGPA are GGOs and consolidation in either a patchy or a predominantly peripheral distribution $(6,19)$. In our study, however, the most common finding in EGPA patients was bronchial wall thickening, which was also considered a cardinal feature of asthma (10), as it was present in $84 \%$ of the asthma patients in our present study. The thickened bronchial wall in HRCT has been related to the subsequent histologic assessments of EGPA that consist of eosinophil infiltration in the mucosa, epithelial basement membrane 


\section{Page 10 of 12}

thickening, smooth muscle hypertrophy, and bronchial stenosis (11). In our study, air trapping in expiratory CT was also observed in 58 of 59 examined participants. Both bronchial wall thickening and air trapping were significantly more common in EGPA patients than in severe asthmatics, indicating that small- and middle-sized airways are more frequently or more severely impaired in EGPA than in severe asthma. Silva et al. reported eosinophilic infiltration in the airway wall with other characteristics including necrotizing infiltration in the airway wall and bronchial granuloma (11). Air trapping on CT scans as a feature of small airway obstructive lesions has not been previously reported in EGPA. The air-trapping sign may play a role in the differential diagnosis of EGPA and similar diseases without small airway obstructions, such as chronic eosinophilic pneumonia and parasitic infections. These diseases frequently present with increased blood eosinophils, but not with air trapping (8).

Another common HRCT feature in participants with EGPA in our study was the presence of increased small vascular markings, which were found in $46 \%$ of EGPA participants compared with only $2 \%$ of those with severe asthma. Increased small vascular markings specifically refer to dilated pulmonary arterioles, venules, and capillaries that are no further than $10 \mathrm{~mm}$ into the pleura. An EGPA case with enlarged, irregular, and stellate-shaped arteries has been reported (20); and an increased caliber of peripheral small arterioles with perivascular fluffy opacities has been described in up to $34 \%$ of EGPA patients (6). Both phenomena may be due to pulmonary eosinophil vasculitis and perivascular cellular infiltration.

There have also been reports of interlobular septal thickening in $56-66 \%$ of EGPA patients $(6,21)$, which may be related to the cardiac dysfunction (11). However, we found that only two participants in our series had heart failure.

The HRCT findings described in our study of patients with EGPA localized to the lungs that clearly allow differentiation from those with severe asthma will be clinically useful. Severe asthma has been defined as asthma that requires treatment with high dose inhaled corticosteroids plus a second controller and/or systemic corticosteroids to prevent it from becoming "uncontrolled" or that remains "uncontrolled" despite this therapy (15). However, it is recommended that patients presenting with "difficult asthma" that is asthma that appears to be difficultto-control with existing asthma therapies, should have conditions that mimic asthma excluded. The condition of
Lin et al. Differentiation of EGPA from severe asthma using HRCT

EGPA is one such condition that can present as a case of difficult-to-treat asthma and even as severe asthma (15). The previous recommendation for performing an HRCT scan of the chest in such patients has been only under circumstances when the presentation of asthma is atypical, such as excessive mucus production, rapid decline in lung function, and reduced carbon monoxide gas transfer factor (15). However, the differentiation of severe asthma from EGPA, particularly EGPA that is entirely localized to the lungs, can be more difficult and our study has indicated the necessity of performing an HRCT of the chest to look for parenchymal lesions such as GGO, consolidation, and increased small vascular markings, on a background of bronchial changes that are also a hallmark of severe asthma. The pointers towards a possible diagnosis of EGPA in such patients are entirely laboratory-based, with higher levels of blood eosinophil count and of total serum IgE. Any other clinical suspicion such as extrapulmonary involvement must also motivate the performance of an HRCT scan of the chest. Patients with suspected asthma presenting with treein-bud opacities, bronchial mucus plugging, bronchiectasis, GGOs, consolidation, increased small vascular markings, hypereosinophilia, higher total IgE level, lower FVC (\% predicted), and FEV1/FVC ratio should raise the suspicionincrease the certainty of the diagnosis of EGPA.

There were several limitations to our study. First, while we obtained pathological confirmation in bronchial biopsies from most of the EGPA participants of a vascular eosinophilic inflammation, we did not examine bronchial biopsies from patients with severe asthma for comparison. Second, this was a retrospective study done at the single time-point of the diagnosis of these conditions and we did not follow up these participants, particularly on the HRCT changes, to observe the evolution of the radiological changes. Third, different CT parameters may have influenced the evaluation of some subjective or objective CT findings. Thus, the Sinogram-Affirmed Iterative (SAFIRE) reconstruction algorithm may be helpful to reduce its impact. Fourth, we cannot exclude the effects of treatments as these participants were at various stages of receiving high-dose corticosteroid treatments that may have suppressed the eosinophilic inflammation or vasculitis. Despite these shortcomings, we revealed that HRCT scans are helpful in the diagnosis of EGPA by differentiating its pathological features from those of patients with classical severe asthma. A prospective study is needed to confirm these findings. 


\section{Conclusions}

In summary, bronchial wall thickening and air trapping were common radiologic findings in EGPA patients and severe asthmatics. We also found that chest HRCT scans of EGPA patients showed a greater incidence of tree-in-bud opacities, bronchial mucus plugging, bronchiectasis, GGOs, consolidation, and increased small vascular markings compared with those in severe asthmatics. Patients with suspected asthma presenting with an abnormal parenchymal finding, hypereosinophilia, higher total IgE level, lower FVC (\% predicted), and FEV1/FVC ratio should raise the suspicion of EGPA. Hence, a lung HRCT scan should be considered in patients presenting with severe asthma in whom there is suspicion of EGPA.

\section{Acknowledgments}

Funding: This study was supported by the Natural Science Foundation of Guangdong Province (2019A1515010622); and the Foundation of Featured Clinical Technique of Guangzhou (2019TS24).

\section{Footnote}

Reporting Checklist: The authors have completed the STARD reporting checklist. Available at https://dx.doi. org/10.21037/atm-21-2243

Data Sharing Statement: Available at https://dx.doi. org/10.21037/atm-21-2243

Conflicts of Interest: All authors have completed the ICMJE uniform disclosure form (available at https://dx.doi. org/10.21037/atm-21-2243). The authors have no conflicts of interest to declare.

Ethical Statement: The authors are accountable for all aspects of the work in ensuring that questions related to the accuracy or integrity of any part of the work are appropriately investigated and resolved. A protocol was prepared before the study without registration. Approval of the protocols used in this study was granted by the Institutional Review Board at the First Affiliated Hospital of Guangzhou Medical University (No. 2018035). Written informed consent was retrospectively provided by each participant included in this study. All procedures performed in this study involving human participants were in accordance with the Declaration of Helsinki (as revised in 2013).

Open Access Statement: This is an Open Access article distributed in accordance with the Creative Commons Attribution-NonCommercial-NoDerivs 4.0 International License (CC BY-NC-ND 4.0), which permits the noncommercial replication and distribution of the article with the strict proviso that no changes or edits are made and the original work is properly cited (including links to both the formal publication through the relevant DOI and the license). See: https://creativecommons.org/licenses/by-nc-nd/4.0/.

\section{References}

1. Greco A, Rizzo MI, De Virgilio A, et al. Churg-Strauss syndrome. Autoimmun Rev 2015;14:341-8.

2. Jennette JC, Falk RJ, Bacon PA, et al. 2012 revised International Chapel Hill Consensus Conference Nomenclature of Vasculitides. Arthritis Rheum 2013;65:1-11.

3. Lanham JG, Elkon KB, Pusey CD, et al. Systemic vasculitis with asthma and eosinophilia: a clinical approach to the Churg-Strauss syndrome. Medicine (Baltimore) 1984;63:65-81.

4. Chumbley LC, Harrison EG Jr, DeRemee RA. Allergic granulomatosis and angiitis (Churg-Strauss syndrome). Report and analysis of 30 cases. Mayo Clin Proc 1977;52:477-84.

5. Guillevin L, Guittard T, Bletry O, et al. Systemic necrotizing angiitis with asthma: causes and precipitating factors in 43 cases. Lung 1987;165:165-72.

6. Choi YH, Im JG, Han BK, et al. Thoracic manifestation of Churg-Strauss syndrome: radiologic and clinical findings. Chest 2000;117:117-24.

7. Worthy SA, Müller NL, Hansell DM, et al. Churg-Strauss syndrome: the spectrum of pulmonary CT findings in 17 patients. AJR Am J Roentgenol 1998;170:297-300.

8. Furuiye M, Yoshimura N, Kobayashi A, et al. ChurgStrauss syndrome versus chronic eosinophilic pneumonia on high-resolution computed tomographic findings. J Comput Assist Tomogr 2010;34:19-22.

9. Jayne D. New-generation therapy for ANCA-associated vasculitis. Clin Exp Nephrol 2013;17:694-6.

10. Gupta S, Siddiqui S, Haldar P, et al. Qualitative analysis of high-resolution CT scans in severe asthma. Chest 2009;136:1521-8.

11. Silva CI, Müller NL, Fujimoto K, et al. Churg-Strauss syndrome: high resolution CT and pathologic findings. J 
Thorac Imaging 2005;20:74-80.

12. Nakamoto K, Saraya T, Ogawa Y, et al. Comparison of findings on thoracic computed tomography with the severity and duration of bronchial asthma in patients with eosinophilic granulomatosis with polyangiitis. Respir Med 2018;139:101-5.

13. Nguyen Y, Guillevin L. Eosinophilic granulomatosis with polyangiitis (Churg-Strauss). Semin Respir Crit Care Med 2018;39:471-81.

14. Wolfe F, Smythe HA, Yunus MB, et al. The American College of Rheumatology 1990 Criteria for the Classification of Fibromyalgia. Report of the Multicenter Criteria Committee. Arthritis Rheum 1990;33:160-72.

15. Chung KF, Wenzel SE, Brozek JL, et al. International ERS/ATS guidelines on definition, evaluation and treatment of severe asthma. Eur Respir J 2014;43:343-73. Erratum in: Eur Respir J 2014;43:1216. Erratum in: Eur Respir J 2018;52:1352020.

16. Hansell DM, Bankier AA, MacMahon H, et al. Fleischner Society: glossary of terms for thoracic imaging. Radiology 2008;246:697-722.

Cite this article as: Lin X, Lin Y, Lai Z, Wei S, Qiu M, Li J, Liu Q, Chung KF, Zeng Q, Zhang Q. Retrospective comparison of high-resolution computed tomography of eosinophilic granulomatosis with polyangiitis with severe asthma. Ann Transl Med 2021;9(12):983. doi: 10.21037/atm21-2243
17. Kim YK, Lee KS, Chung MP, et al. Pulmonary involvement in Churg-Strauss syndrome: an analysis of CT, clinical, and pathologic findings. Eur Radiol 2007;17:3157-65.

18. Akira M, Inoue G, Yamamoto S, et al. Non-specific interstitial pneumonia: findings on sequential CT scans of nine patients. Thorax 2000;55:854-9.

19. Fina A, Dubus JC, Tran A, et al. Eosinophilic granulomatosis with polyangiitis in children: data from the French RespiRare ${ }^{\circledR}$ cohort. Pediatr Pulmonol 2018;53:1640-50.

20. Buschman DL, Waldron JA Jr, King TE Jr. ChurgStrauss pulmonary vasculitis. High-resolution computed tomography scanning and pathologic findings. Am Rev Respir Dis 1990;142:458-61.

21. Johkoh T, Müller NL, Akira M, et al. Eosinophilic lung diseases: diagnostic accuracy of thin-section CT in 111 patients. Radiology 2000;216:773-80.

(English Language Editor: J. Jones) 